This document is the accepted manuscript version of the following article:

Danilovich, I. L., Merkulova, A. V., Morozov, I. V., Ovchenkov, E. A., Spiridonov, F. M., Zvereva, E. A., ... Vasiliev, A. N. (2019). Strongly canted antiferromagnetic ground state in $\mathrm{Cu} 3(\mathrm{OH}) 2 \mathrm{~F} 4$. Journal of Alloys and Compounds, 776, 16-21. https://doi.org/10.1016/j.ja11com.2018.10.032

This manuscript version is made available under the CC-BY-NC-ND 4.0 1icense http://creativecommons.org/1icenses/by-nc-nd/4.0/

\title{
Strongly Canted Antiferromagnetic Ground State in $\mathrm{Cu}_{3}(\mathrm{OH})_{2} \mathrm{~F}_{4}$
}

Igor L. Danilovich, ${ }^{\dagger}$ Anna V. Merkulova, ${ }^{\dagger}$ Igor V. Morozov, ${ }^{\dagger}$ Evgeniy A. Ovchenkov, ${ }^{\dagger}$ Felix M. Spiridonov, ${ }^{\dagger}$ Elena A. Zvereva, ${ }^{\dagger} \pitchfork$ Olga S. Volkova, ${ }^{\dagger}, \S$ Vladimir V. Mazurenko, ${ }^{\S}$ Zlata V. Pchelkina, $,^{\S},{ }^{\perp}$ Alexander A. Tsirlin, $\$, \|$ Christian Balz, ${ }^{\#}$ Stefan Holenstein,\%,@ Hubertus Luetkens, ${ }^{\%}$ Alexander A. Shakin, ${ }^{\$}$ Alexander N. Vasiliev $* \dot{t}, \mathbf{w} \$$

${ }^{\dagger}$ Moscow State University, Moscow 119991, Russia

National Research South Ural State University, Chelyabinsk, 454080, Russia

\$Ural Federal University, Ekaterinburg 620002, Russia

${ }^{\perp}$ Institute of Metal Physics, RAS, Ekaterinburg 620137, Russia

\|University of Augsburg, Augsburg 86135, Germany

${ }^{\#}$ Helmholtz-Zentrum Berlin for Materials and Energy, Berlin 14109, Germany

${ }^{\%}$ Paul Scherrer Institute, Villigen 5232, Switzerland

${ }^{\circledR}$ Universität Zürich, Zurich 8057, Switzerland

\$National University of Science and Technology "MISiS", Moscow 119049, Russia

ABSTRACT: An unique crystal structure of copper hydroxyl-fluorite, $\mathrm{Cu}_{3}(\mathrm{OH})_{2} \mathrm{~F}_{4}$, hosts the trimerized chains of both edge-sharing and corner-sharing $\mathrm{CuO}_{2} \mathrm{~F}_{2}$ plaquettes. The results of the comprehensive study of this compound, including new synthetic route, measurements of specific heat, $a c$ - and $d c$-susceptibility, pulsed field magnetization, electron spin resonance, muon spin rotation and relaxation and first principles calculations are presented. The data evidence magnetic phase transition at $T_{\mathrm{C}}=12.5 \mathrm{~K}$ into canted antiferromagnetic state which is due to antisymmetric Dzyaloshinskii-Moriya (DM) exchange interaction. No alteration of DM component stemming from the intrinsic features of the crystal lattice in $\mathrm{Cu}_{3}(\mathrm{OH})_{2} \mathrm{~F}_{4}$ results in unusually large spontaneous magnetization. At $T<T_{\mathrm{C}}$, the remanence $M_{\mathrm{R}}$ constitutes significant portion of saturation magnetization $M_{\mathrm{S}}$ which defines the canting angle $\varphi=4^{\circ}$. 


\section{INTRODUCTION}

The mixed-anion salts of 3d-metals offer vast playground to investigate the relationships between structure and properties in magnetic systems. The interest to this multitude is due to the fact that both physics and chemistry of mixed-anion compounds significantly differ from those in single-anion ones. The "simplest" in this family are the objects where the cation subsystem is represented by single transition metal and the anion subsystem is constituted by several entities, e.g. $(\mathrm{OH})^{-}, \mathrm{F}^{-}, \mathrm{Cl}^{-}, \mathrm{Br}^{-}$and/or $\mathrm{O}^{2-}$. The physics of mixed-anion salts of transition metals is less studied since many of these objects are sensitive to moisture and decompose at relatively low temperatures.

Among physical properties the most sensitive to chemical composition are the magnetic ones since very diverse is the overlap of transition metal d-orbitals with oxygen, halogen or hydroxyl orbitals. This results in pronounced dispersion and competition of various exchange interactions in mixed-anion compounds. At the same time, in such cases no exchange pathways are present with participation of other transition and non-transition cations which simplifies the analysis of exchange interactions. The basic families of $3 \mathrm{~d}$-metals polyanion compounds are the oxyhalides $\operatorname{MOX}(\mathrm{M}=\mathrm{Sc}-\mathrm{Cr}$ and $\mathrm{Fe} ; \mathrm{X}=\mathrm{Cl}$ and $\mathrm{Br})[1-5]$, the hydroxyhalides $\mathrm{M}(\mathrm{OH}) \mathrm{X}(\mathrm{M}=\mathrm{Co}$ and $\mathrm{Cu}$; $\mathrm{X}=\mathrm{F}$ and $\mathrm{Cl})$ [6-8] and the trihydroxyhalides $\mathrm{M}_{2}(\mathrm{OH})_{3} \mathrm{X}(\mathrm{M}=\mathrm{Mn}-\mathrm{Cu} ; \mathrm{X}=\mathrm{Cl}$ and $\mathrm{Br})$ [9-11].

The members of oxyhalides family, $\mathrm{TiOCl}$ and $\mathrm{TiOBr}$, are known for nonconventional spinPeierls-like instability [12-14]. Similarly strong magnetoelastic coupling accompanied by the doubling of crystal lattice parameters at Neel temperature was observed in $\mathrm{VOCl}[15,16], \mathrm{CrOCl}$ [17], and $\mathrm{FeOCl}[18]$. The Shastry-Sutherland magnetic network is the interesting feature of quasi-two-dimensional hydroxyhalide $\mathrm{Cu}(\mathrm{OH}) \mathrm{Cl}$ which orders antiferromagnetically at low temperatures [19]. Some 3d-metals trihydrohalides are characterized by heavy frustration of exchange interactions which suppresses significantly the formation of the long-range magnetic order [20-23]. Besides, the minerals barlowite $\mathrm{Cu}_{4}(\mathrm{OH})_{6} \mathrm{FBr}$ and melanothallite $\mathrm{Cu}_{2} \mathrm{OCl}_{2}$ can be also classified as belonging to cupric mixed-anion compounds. The former attracts attention due to its canted antiferromagnetic ground state with $\mathrm{Cu}^{2+}$ ions in perfect kagome arrangement [24] while the latter is studied due to its high-temperature multiferroic properties [25].

Here, we present the results of both experimental and theoretical investigation of copper hydroxyl-fluorite, $\mathrm{Cu}_{3}(\mathrm{OH})_{2} \mathrm{~F}_{4}$, including synthesis, measurements of various thermodynamic and resonance properties and first principles calculations. Except Raman spectra taken at room 
temperature no study of physical properties of the title compound was reported so far [26]. Preliminary results of magnetic characterization of $\mathrm{Cu}_{3}(\mathrm{OH})_{2} \mathrm{~F}_{4}$ were included into the review on magnetism in mixed-anion compounds of transition metals [27].

\section{EXPERIMENTAL METHODS AND CALCULATION DETAILS}

Thermodynamic properties, i.e. ac- and dc-susceptibility $\chi$ and specific heat $C$ of $\mathrm{Cu}_{3}(\mathrm{OH})_{2} \mathrm{~F}_{4}$ in the temperature range $2 \div 400 \mathrm{~K}$ under magnetic field up to $B=9 \mathrm{~T}$ were measured using various options of "Quantum design" MPMS-7T and PPMS-9T devices. The hysteresis loops were taken at various temperatures under magnetic field up to $1 \mathrm{~T}$. Pulsed magnetic field measurements up to $B=30 \mathrm{~T}$ were performed using home-made inductive coil set-up with pulse rise-time $t \sim 8 \mathrm{msec}$. The electron spin resonance study of powder sample of $\mathrm{Cu}_{3}(\mathrm{OH})_{2} \mathrm{~F}_{4}$ was performed using an X-band ESR spectrometer CMS 8400 (ADANI) $(f \sim 9.4 \mathrm{GHz}, B \leq 0.7 \mathrm{~T})$ equipped with a low temperature mount, operating in the range $T=6 \div 300 \mathrm{~K}$. The effective $g$ factor has been calculated with respect to a BDPA (a,g -bisdiphenyline-b-phenylallyl) reference sample with $g$-factor $g=2.00359$. Zero field muon spin rotation and relaxation $(\mathrm{ZF}-\mu \mathrm{SR})$ were measured at GPS, Swiss Muon Source at the Paul Scherrer Institute in a temperature range between $40 \mathrm{~K}$ and $1.5 \mathrm{~K}$. A $500 \mathrm{mg}$ pressed powder pellet of $\mathrm{Cu}_{3}(\mathrm{OH})_{2} \mathrm{~F}_{4}$ was wrapped in aluminum foil to protect it from moisture and mounted on a standard sample holder. The data were analyzed using the musrfit software package [28].

The electronic structure of $\mathrm{Cu}_{3}(\mathrm{OH})_{2} \mathrm{~F}_{4}$ was calculated within the density functional theory using the Quantum ESPRESSO software package [29]. The exchange-correlation part of the total energy functional was used in the PBE approximation [30]. A kinetic energy cutoff for the planewave expansion of the electronic states was set to $80 \mathrm{Ry}$. Reciprocal space integration were done on a regular $4 \times 6 \times 2 \mathrm{k}$-points grid in the irreducible part of the Brillouin zone.

The direct calculation of exchange coupling constants within the projector augmented wave (PAW) method as implemented in Vienna ab initio simulation package (VASP) [31] was also performed. Integration in the reciprocal space was done on a regular $4 \times 4 \times 4 \mathrm{k}$-points mesh in the irreducible part of Brillouin zone. The energy cutoff value for plane wave functions expansion equals $400 \mathrm{eV}$. The values of isotropic exchange constants were obtained from the total energies of different spin configurations using four-configuration approach [32]. The calculations were 
done in the framework of GGA approximation with rotationally invariant form of $U$ correction [33].

\section{RESULTS AND DISCUSSION}

3.1. Synthesis, crystal structure and magnetic interaction pathways

The copper hydroxyl-fluorite, $\mathrm{Cu}_{3}(\mathrm{OH})_{2} \mathrm{~F}_{4}$, was firstly obtained as a by-product of natrochalcite-type compound syntheses [26]. Here, we suggest the synthetic approach for obtaining single phase of $\mathrm{Cu}_{3}(\mathrm{OH})_{2} \mathrm{~F}_{4}$. The sample of $\mathrm{Cu}_{3}(\mathrm{OH})_{2} \mathrm{~F}_{4}$ was obtained as an olive green powder by heating the mixture of $\mathrm{CuF}_{2} \times 2 \mathrm{H}_{2} \mathrm{O}$ and $\mathrm{Cu}(\mathrm{OH}) \mathrm{F}$ powders in the ratio $1: 2$ in a sealed Teflon-lined steel vessel under $200{ }^{\circ} \mathrm{C}$ for two days. The resulting product is not stable in air due to moisture adsorption.

The correspondence of crystal structure of newly synthesized sample to that defined earlier [26] was confirmed by powder X-ray diffraction measurements at room temperature.

$\mathrm{Cu}_{3}(\mathrm{OH})_{2} \mathrm{~F}_{4}$ crystallizes in monoclinic $P 2_{1} / n$ space group, $Z=2, a=5.494(5) \AA, b=4.744(4) \AA$, $c=8.436(8) \AA, \beta=92.22(4)^{\circ}$, and $V=219.7(5) \AA^{3}$.

To the best of authors' knowledge, no structures isotypic to $\mathrm{Cu}_{3}(\mathrm{OH})_{2} \mathrm{~F}_{4}$ were reported. There are two distinct sites for $\mathrm{Cu}$ atoms in two $\mathrm{Cu} 2$ to one $\mathrm{Cu} 1$ ratio both in $4+2$ coordination, the equatorial $\mathrm{CuO}_{2} \mathrm{~F}_{2}$ plaquettes being formed by two $\mathrm{O}$ and $\mathrm{F}$ atoms, each. The structure of $\mathrm{Cu}_{3}(\mathrm{OH})_{2} \mathrm{~F}_{4}$ is characterized by trimerized chains of edge- and corner-sharing $\mathrm{Cu}(\mathrm{OH})_{2} \mathrm{~F}_{4}$ polyhedra running along the [011] axis, which are linked to each other via corners to form a three-dimensional network, as shown in the left panel of Figure 1. Within the $\mathrm{Cu} 2-\mathrm{Cu} 1-\mathrm{Cu} 2$ trimerized chains the $\mathrm{Cu} 1-\mathrm{F}-\mathrm{Cu} 2$ angle is $130.2^{\circ}$ while the $\mathrm{Cu} 1-\mathrm{O}-\mathrm{Cu} 1$ angle is $98.4^{\circ}$. According to Goodenough-Kanamori rules [34,35], the latter angle is close to critical angle of compensation of ferromagnetic and antiferromagnetic contributions to superexchange interaction. The main exchange pathways $J_{1}-J_{6}$ in $\mathrm{Cu}_{3}(\mathrm{OH})_{2} \mathrm{~F}_{4}$ are depicted in the right panel of Figure 1. 


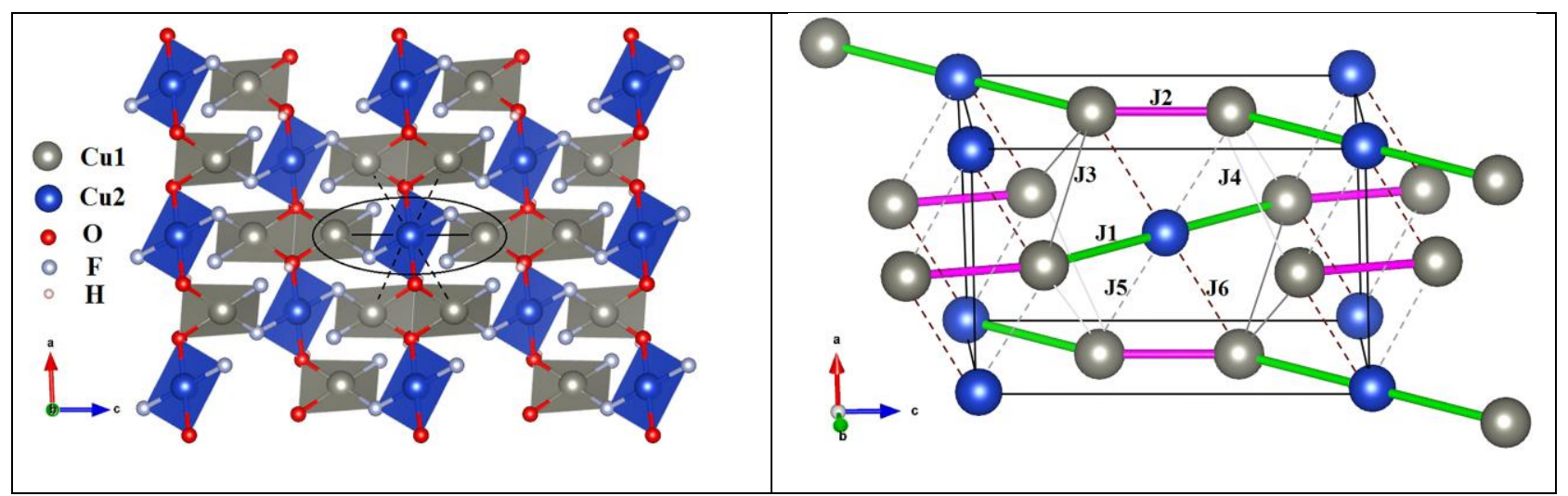

Figure 1. Left panel. The crystal structure of $\mathrm{Cu}_{3}(\mathrm{OH})_{2} \mathrm{~F}_{4}$ in polyhedral representation. The intrachain and interchain exchange pathways are shown by solid and dashed lines, respectively. The trimer unit is marked by the oval. Right panel. The scheme of exchange interactions within unit cell of $\mathrm{Cu}_{3}(\mathrm{OH})_{2} \mathrm{~F}_{4}$, the thick solid lines represents intratrimer $J 1$ and intertrimer $J 2$ intrachain exchange interactions, the thin solid and dash lines represents various interchain exchange inter-trimer interactions $\overparen{3}-J 6$.

\subsection{Electron spin resonance}

Electron spin resonance (ESR) powder pattern in $\mathrm{Cu}_{3}(\mathrm{OH})_{2} \mathrm{~F}_{4}$ is characteristic of $\mathrm{Cu}^{2+}$ ions with an anisotropic $g$-tensor. The amplitude of the signal increases monotonously upon cooling down to $\sim 12.5 \mathrm{~K}$. At this temperature, the signal degrades which points to the opening of a spin gap for resonance excitations due to the onset of a long range magnetic order. To evaluate the main ESR parameters the experimental spectra were fitted by the sum of three components corresponding to the principal values of g-tensor using sum of three Lorentzian profile, which has been taken in a conventional form:

$$
\frac{d P}{d B} \propto \frac{d}{d B}\left[\frac{\Delta B}{\left(B-B_{r}\right)^{2}+\Delta B^{2}}\right],
$$

where $P$ is the power absorbed in the ESR experiment, $B_{r}$ is the resonance field and $\Delta B$ is the linewidth. A representative example of the lineshape analysis is given in the upper panel of Figure 2 where the resolved resonance modes are denoted by dashed lines and their sum is shown by the solid line. The presence of three well resolved components indicates low symmetry of the crystal environment and is typical for $\mathrm{Cu}^{2+}$ in distorted square planar coordination [36,37]. 
The temperature dependences of linewidths $\Delta B$ and effective $g$-factors in $\mathrm{Cu}_{3}(\mathrm{OH})_{2} \mathrm{~F}_{4}$ are shown in the middle and lower panels of Figure 2. The principal $g$-values of the anisotropic g-tensor remain temperature independent over the whole temperature range studied with the values $g_{1}=2.42 \pm 0.02, g_{2}=2.25 \pm 0.02$ and $g_{3}=2.13 \pm 0.02$. The averaged value $g=2.27 \pm 0.02$ is consistent with typical values for $\mathrm{Cu}^{2+}$ ions in other copper oxides. The ESR linewidths demonstrate slight narrowing with decreasing temperature. Below $\sim 50 \mathrm{~K}$, the deviation of linewidths $\Delta B$ and $g$ factors from the room-temperature values indicates the development of internal magnetic fields upon approach to long range ordered phase.
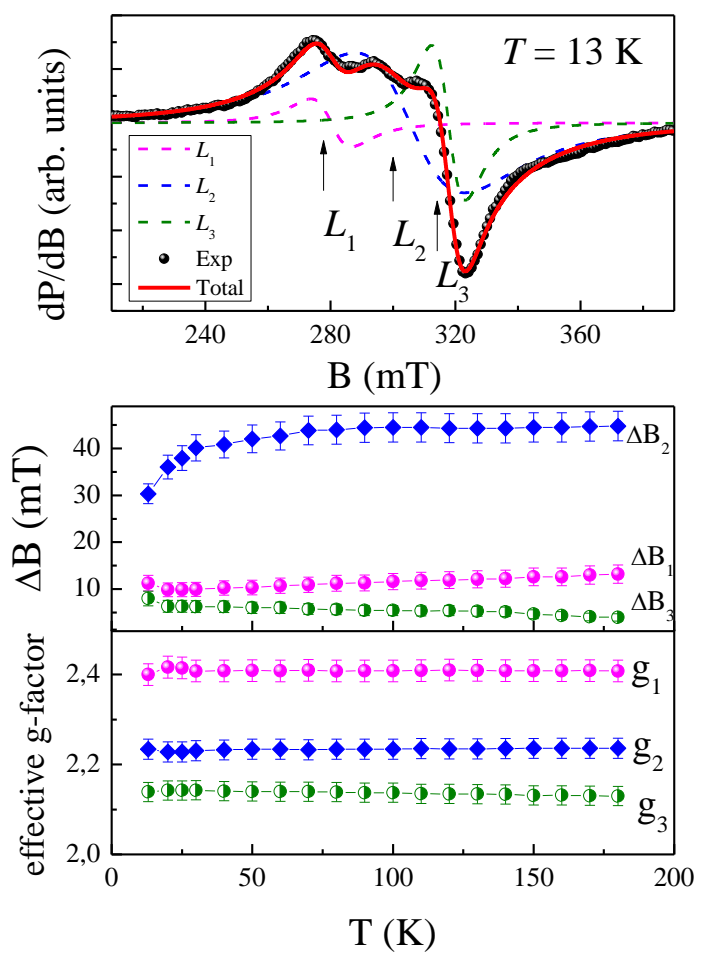

Figure 2. Upper panel. The low temperature ESR spectrum in $\mathrm{Cu}_{3}(\mathrm{OH})_{2} \mathrm{~F}_{4}$ at $\mathrm{T}>\mathrm{T}_{\mathrm{C}}$. Symbols are experimental data, the dashed lines show the individual Lorentzian fit components and the solid line is the sum of all three. Lower panel. The temperature dependences of the linewidths $\Delta \mathrm{B}$ and g-factors of the principal components of the g-tensor for three resolved modes of the ESR spectra. 


\subsection{Thermodynamic properties}

The temperature dependences of both real $\chi^{\prime}$ and imaginary $\chi^{\prime \prime}$ components of $a c$-magnetic susceptibility taken at the frequency $f=10 \mathrm{~Hz}$ and magnetic field $h=10 \mathrm{Oe}$ in $\mathrm{Cu}_{3}(\mathrm{OH})_{2} \mathrm{~F}_{4}$ are shown in the left panel of Figure 3. Very sharp singularities in both components at $T_{\mathrm{C}}=12.5 \mathrm{~K}$ mark the onset of long range magnetic order. No shift of transition temperature was detected in the frequency range $10 \div 10^{4} \mathrm{~Hz}$, signaling high quality of the sample and meaning no sizable spin-glass or disorder effects. The inset to the left panel of Figure 3 represents the temperature dependence of the specific heat $C$. The phase transition is marked by an anomaly which is broad tentatively due to poor thermal contact between the grains of powder sample. The entropy released below $T_{\mathrm{C}}$ equals to $4.2 \mathrm{~J} / \mathrm{molK}$ being less than a quarter of total magnetic entropy $S_{\text {magn }}$ $=3 R \ln 2=17.3 \mathrm{~J} / \mathrm{molK}$ expected for this system. The release of substantial entropy above $T_{\mathrm{C}}$ could be considered as an evidence of reduced dimensionality and frustration in the magnetic subsystem of $\mathrm{Cu}_{3}(\mathrm{OH})_{2} \mathrm{~F}_{4}$.

The temperature dependence of $d c$-susceptibility $\chi$ taken at $B=0.1 \mathrm{~T}_{\text {in }} \mathrm{Cu}_{3}(\mathrm{OH})_{2} \mathrm{~F}_{4}$ is shown in the middle panel of Figure 3. Up to $390 \mathrm{~K}$ it never follows the Curie-Weiss law. The temperature dependence of inverse $d c$-susceptibility $\chi^{-1}$ in $\mathrm{Cu}_{3}(\mathrm{OH})_{2} \mathrm{~F}_{4}$ is shown in the inset to the middle panel of Figure 3. In accordance with the crystal structure motif this dependence can be fitted by expression for isolated magnetic trimers [38]

$$
\chi^{-1}(T)=\frac{4 k_{B} T}{N_{A} g^{2} \mu_{B}^{2}} \frac{2 e^{\left(-\frac{J_{1}}{2 k_{B} T}\right)}+e^{\left(\frac{J_{1}}{k_{B} T}\right)}+1}{10 e^{\left(-\frac{J_{1}}{2 k_{B} T}\right)}+e^{\left(\frac{1}{k_{B} T}\right)}+1},
$$

where the next-nearest-neighbor exchange interaction within the trimer is neglected and $k_{\mathrm{B}}, N_{\mathrm{A}}$ and $\mu_{\mathrm{B}}$ are Boltzmann, Avogadro and Bohr constants. The fitting with averaged g-factor of $\mathrm{Cu}^{2+}$ ions $g=2.27$ results in the intra-trimer exchange interaction parameter $\mathrm{J}_{1} \sim 140 \mathrm{~K}$. The exact value depends somewhat on the fitting range. 

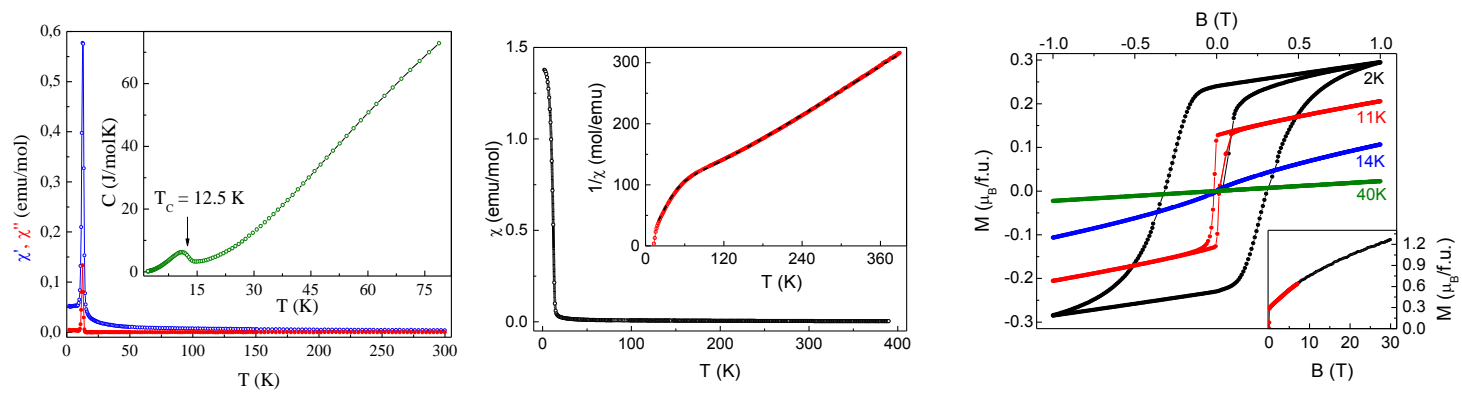

Figure 3. Left panel. The temperature dependences of real $\chi^{\prime}$ and imaginary $\chi^{\prime \prime}$ components of ac-magnetic susceptibility at $f=10 \mathrm{~Hz}$ in $\mathrm{Cu}_{3}(\mathrm{OH})_{2} \mathrm{~F}_{4}$. The inset represents the temperature dependence of specific heat $C_{\mathrm{p}}$. Middle panel. The temperature dependence of dc-magnetic susceptibility in $\mathrm{Cu}_{3}(\mathrm{OH})_{2} \mathrm{~F}_{4}$ taken at $\mathrm{B}=0.1 \mathrm{~T}$. The inset represents inverse magnetic susceptibility $\chi^{-1}(T)$. The fitting curve at $T>T_{\mathrm{C}}$ is shown by the dashed line. Right panel. The hysteresis loops in $\mathrm{Cu}_{3}(\mathrm{OH})_{2} \mathrm{~F}_{4}$ taken at various temperatures both above and below transition temperature $T_{\mathrm{C}}=12.5 \mathrm{~K}$. The inset represents $M(B)$ curve taken in pulsed magnetic fields.

The step-like anomaly in $\chi(T)$ curve at $T_{\mathrm{C}}=12.5 \mathrm{~K}$ in $\mathrm{Cu}_{3}(\mathrm{OH})_{2} \mathrm{~F}_{4}$ is the signature of the long range order with a spontaneous magnetic moment $M$. The field dependences of magnetization, shown in the right panel of Figure 3, define the remanence $M_{\mathrm{R}}=0.24 \mu_{\mathrm{B}} /$ f.u. and the coercivity $B_{\mathrm{C}}=0.32 \mathrm{~T}$ of hysteresis loop at lowest temperature. The $M(B)$ dependence taken at $2 \mathrm{~K}$ in pulsed magnetic field up to $30 \mathrm{~T}$ is shown in the inset to the right panel of Figure 3. This curve was calibrated by measurements of magnetization in static magnetic fields up to 7T. The moment at highest magnetic fields exceeds $\sim 1.25 \mu_{\mathrm{B}} / \mathrm{f}$.u. which is slightly higher than $1 / 3$ of expected saturation magnetization $\mathrm{M}_{\mathrm{S}} \sim 3.4 \mu_{\mathrm{B}} / \mathrm{f}$.u. At low temperatures, the remanence $M_{\mathrm{R}}$ constitutes significant portion of saturation magnetization $M_{\mathrm{S}}$. This feature points to a strongly canted antiferromagnetic ground state in $\mathrm{Cu}_{3}(\mathrm{OH})_{2} \mathrm{~F}_{4}$ with the canting angle $\varphi=4^{\circ}$ defined through $\sin \varphi$ $=M_{\mathrm{R}} / M_{\mathrm{S}}$.

\subsection{Muon spin relaxation}

To obtain further insight into the magnetic phase transition of $\mathrm{Cu}_{3}(\mathrm{OH})_{2} \mathrm{~F}_{4}$ found by magnetic susceptibility and heat capacity, zero field muon spin relaxation, $\mathrm{ZF}-\mu \mathrm{SR}$, measurements were performed. Representative spectra are shown in the left panel of Figure 4. Clearly different muon spin polarization $P(t)$ curves are observed above and below $T_{\mathrm{C}}$, as defined in measurements of 
magnetic susceptibility $\chi$ and specific heat $C$. Above $T_{\mathrm{C}}, P(t)$ shows weak relaxation consistent with an F- $\mu-\mathrm{F}$ bound state expected for materials containing $\mathrm{F}^{-}$ions [39]. Below $T_{\mathrm{C}}$, spontaneous coherent muon spin precession is observed indicating the development of static magnetic fields at the muon sites. The spectra could only be fitted assuming two magnetically inequivalent muon stopping sites in $\mathrm{Cu}_{3}(\mathrm{OH})_{2} \mathrm{~F}_{4}$. Above $T_{\mathrm{C}}$, the spectra were modelled with a damped Gaussian Kubo-Toyabe function to ascribe for the weak relaxation [40]. Therefore, the following polarization function was used to describe the ZF- $\mu$ SR data:

$$
\begin{aligned}
& P(t)=f_{\text {mag }} P_{\text {mag }}(t)+\left(1-f_{\text {mag }}\right) P_{N M}(t)+B k g \\
& \text { with } P_{N M}(t)=e^{-\lambda_{d y n}} G K T(t) \\
& \text { and } P_{\text {mag }}(t)=\frac{2}{3}\left(0.5 \cos \left(\gamma B_{1} t\right) e^{-\frac{1}{2}\left(\sigma_{1} t\right)^{2}}+0.5 \cos \left(\gamma B_{2} t\right) e^{-\frac{1}{2}\left(\sigma_{2} t\right)^{2}}\right)+\frac{1}{3} e^{-\lambda_{d y n} t} .
\end{aligned}
$$

Here, $f_{\text {magn }}$ is the magnetic volume fraction, $\lambda_{\text {dyn }}$ is the dynamic relaxation rate, and GKT is the Gaussian Kubo-Toyabe function. In the magnetic fraction, $\gamma$ is the gyromagnetic ratio of the muon, $B_{1,2}$ are the internal fields at the muon sites, and $\sigma_{1,2}$ are the transverse relaxation rates. The sum of a $2 / 3$ and a $1 / 3$ fraction originates from the powder average of the internal magnetic fields with respect to the initial muon spin polarization. For a polycrystalline sample, $2 / 3$ of the internal field components are perpendicular to the muon spin while 1/3 are parallel. The relaxation $\sigma_{1,2}$ of the perpendicular (transverse) component is due to the finite width of the static field distribution and potentially dynamic processes while the parallel (longitudinal) relaxation $\lambda_{\text {dyn }}$ stems from dynamic processes only. The ratio of the internal magnetic fields $B_{1,2}$ at the two muon sites was found to remain constant as a function of temperature and has been fixed to the low temperature fit of $B_{1}=0.573 B_{2}$. This is consistent with observing the same magnetic phenomena from two different perspectives. The relatively strong suppression of the oscillations as shown in the left panel of Figure 4 indicates a rather short magnetic correlation lengths which may be caused by crystal defects or disorder in the anion distribution in $\mathrm{Cu}_{3}(\mathrm{OH})_{2} \mathrm{~F}_{4}$. In addition to these two fractions, a fraction of $B k g=11 \%$ is found to be non-relaxing and is ascribed to muons not stopping in the sample. This background fraction is temperature independent. 

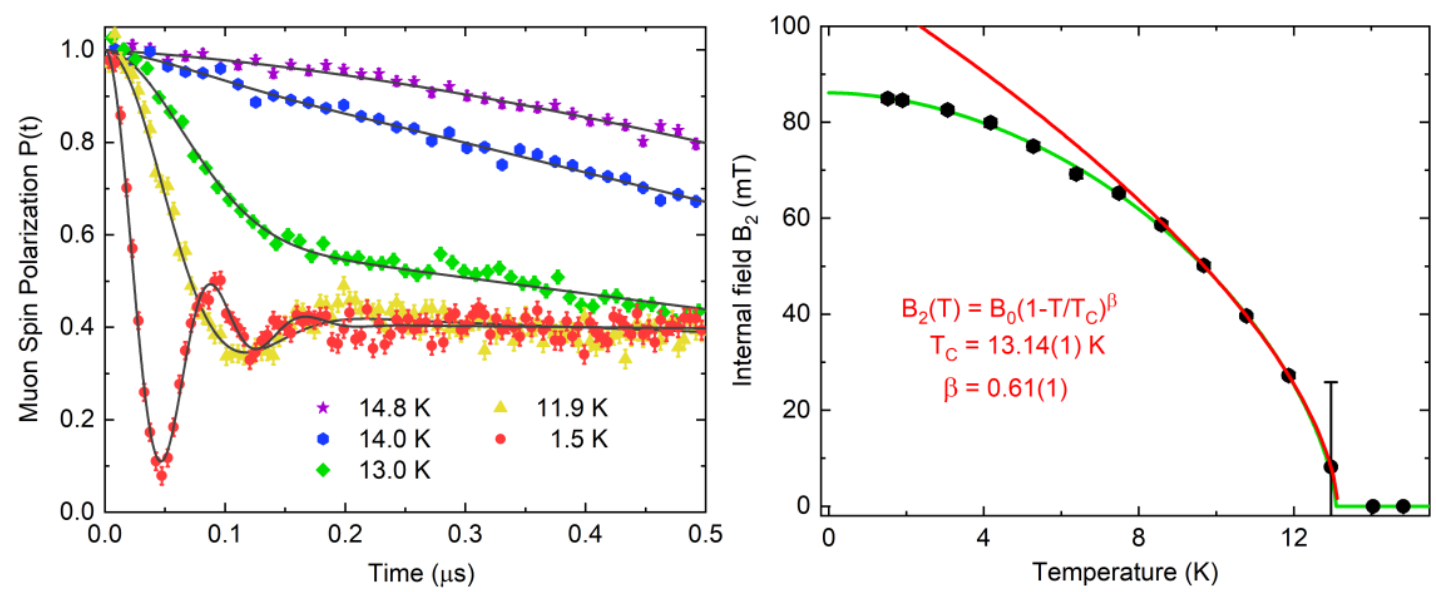

Figure 4. Left panel. Zero-field muon spin polarization spectra of $\mathrm{Cu} 3(\mathrm{OH}) 2 \mathrm{~F} 4$. The oscillations at $\mathrm{T}<\mathrm{TC}$ indicate the onset of long-range magnetic order. The spectra are fitted to a two muon sites model. Right panel. Development of the internal magnetic field in $\mathrm{Cu} 3(\mathrm{OH}) 2 \mathrm{~F} 4$ at muon site 2 equivalent to the magnetic order parameter. The green line is a guide to the eye. The red line is a fit to the power law shown in the figure for data above $9.5 \mathrm{~K}$.

The results from fitting the ZF- $\mu$ SR spectra to Eq. (3) are shown in the right panel of Figure 4. A gradual increase of internal magnetic fields is observed below $T_{\mathrm{C}}$ which describes the magnetic order parameter in $\mathrm{Cu}_{3}(\mathrm{OH})_{2} \mathrm{~F}_{4}$. In addition, a sharp maximum in the spin lattice relaxation rate $\lambda_{\text {dyn }}$ is observed at $T_{\mathrm{C}}$ confirming a second-order magnetic phase transition. The magnetic volume fraction increases rapidly to $100 \%$ at the phase transition confirming that the full sample volume orders magnetically. Fitting the internal magnetic field evolution to the power law $B_{2}=B_{0}\left(1-T / T_{\mathrm{C}}\right)^{\beta}$ one obtains the phase transition temperature $T_{\mathrm{C}}$ and the critical exponent $\beta$. Using data above $9.5 \mathrm{~K}$ one gets $T_{\mathrm{C}}=13.1 \mathrm{~K}$ and $\beta=0.61$. The transition temperature is roughly consistent with the one obtained from bulk measurements while being slightly higher. The critical exponent cannot reliably be determined due to the lack of high density data points close to the phase transition.

\subsection{First principles calculations}

Non-spin-polarized self-consistent calculation has been carried out for the title compound. The band structure obtained within generalized gradient approximation (GGA) is presented in Figure 5 by black lines. We note that GGA predicts $\mathrm{Cu}_{3}(\mathrm{OH})_{2} \mathrm{~F}_{4}$ to be metallic. It is well known that GGA fails to describe the correct insulating ground state for strongly correlated electron system, 
as is the case here. The inclusion of the missing correlation effect, as mentioned, beyond GGA in a partially filled $\mathrm{Cu}-\mathrm{d}$ manifold provides the insulating description of the system. We have checked this by treating the correlation within the GGA $+U$ framework. Although GGA fails to provide the correct ground state for this class of materials, it describes the bonding and chemistry aspects correctly.

In order to calculate the hopping integrals between $\mathrm{Cu}$ sites the Wannier function projection on $\mathrm{Cu} d_{x^{2}-y^{2}}$-like orbitals was applied using Wannier90 code [41]. The $\mathrm{Cu}$ bands after projection are shown by red lines in Figure 5. The main exchange paths for $\mathrm{Cu}_{3}(\mathrm{OH})_{2} \mathrm{~F}_{4}$ are shown in the right panel to Figure 1. The corresponding hoppings are presented in the third column in Table I. The trimer motif is formed by $\mathrm{Cu} 2-\mathrm{Cu} 1-\mathrm{Cu} 2$ ions connected by exchange coupling $J_{l}$. The primary hopping is the one within the trimer. The estimation of antiferromagnetic contribution to

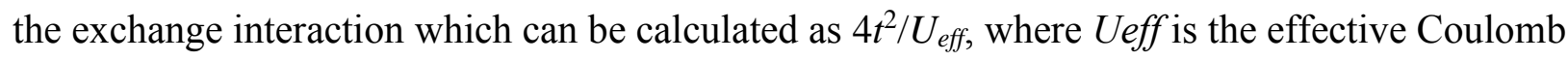
repulsion taken to be $6 \mathrm{eV}$, is listed in the fourth column in Table 1.

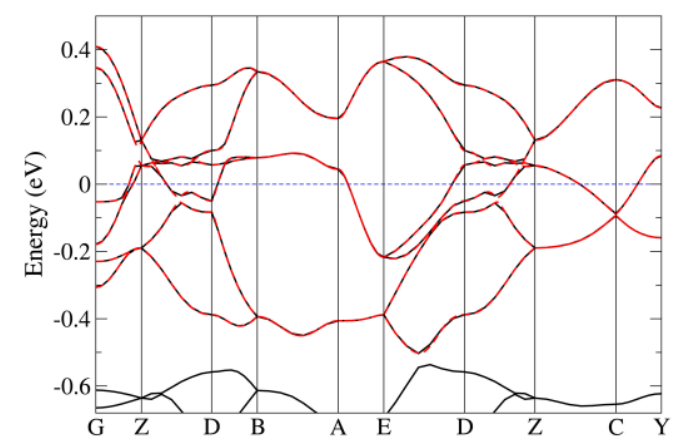

Figure 5. GGA band structure of $\mathrm{Cu}_{3}(\mathrm{OH})_{2} \mathrm{~F}_{4}$ (black curves). Bands obtained by Wannier

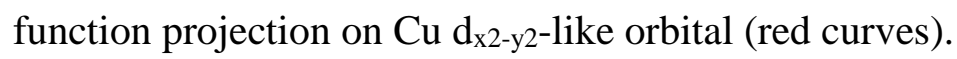


Table 1. Hopping integrals $t$ obtained in GGA calculations by Wannier function projection (in $\mathrm{meV}$ ), AFM contribution to the exchange $J^{\mathrm{AFM}}$ (in $\mathrm{K}$ ) estimated according to the expression $4 t^{2} / U_{\text {eff }}$ with $U_{\text {eff }}=6 \mathrm{eV}$, and exchange integrals $J$ (in $\mathrm{K}$ ) calculated within VASP (the positive sigh of exchange integrals corresponds to antiferromagnetic interaction).

\begin{tabular}{lllll}
\hline Label & Distance, A & $t$, mev & $J^{\mathrm{AFM}}, \mathrm{K}$ & $J, \mathrm{~K}$ \\
$J_{1}$ & 3.52 & 137 & 145 & 142 \\
$J_{2}$ & 3.00 & 127 & 125 & 11 \\
$J_{3}$ & 3.12 & 74 & 42 & 8 \\
$J_{4}$ & 3.14 & -77 & 46 & 14 \\
$J_{5}$ & 3.74 & & & 12 \\
$J_{6}$ & 3.95 & & & 12 \\
\hline
\end{tabular}

The direct calculation of exchange coupling constants was performed using the onsite Coulomb repulsion parameter $U=9.5 \mathrm{eV}$ and the intra-atomic Hund's rule exchange interaction $J_{H}=0.9 \mathrm{eV}$. The calculated exchange integrals are antiferromagnetic and presented in the last column of Table I. One can see that the strongest exchange interaction $J_{l}=142 \mathrm{~K}$ is within trimer. The antiferromagnetic contribution to the exchange $J_{2}$ between trimers is strongly compensated resulting in $J_{2}=11 \mathrm{~K}$, which is thirteen times smaller than $J_{l}$. The inverse magnetic susceptibility simulated within trimer model with $J_{I}=142 \mathrm{~K}$ is shown in the inset to middle panel of Figure 3.

\section{CONCLUSION}

An experimental study of $\mathrm{Cu}_{3}(\mathrm{OH})_{2} \mathrm{~F}_{4}$, including measurements of specific heat, $a c$ - and $d c$ susceptibility, pulsed field magnetization, electron spin resonance, muon spin rotation and relaxation evidence magnetic phase transition at $T_{\mathrm{C}}=12.5 \mathrm{~K}$ into canted antiferromagnetic state. The first principles calculations allows estimating the full set of exchange interaction parameters, all of them appear to be antiferromagnetic. 
The $\mathrm{Cu}$ sites in $\mathrm{Cu}_{3}(\mathrm{OH})_{2} \mathrm{~F}_{4}$ are coupled by various exchange interactions. This interaction is very large within trimeric units $(J 1 \sim 140 \mathrm{~K})$, but the magnetic order establishes only when significantly weaker exchange interactions $(J 2-J 6 \sim 12 \mathrm{~K})$ come into play and orient the trimeric units with respect to each other. The weakness of $J 2-J 6$ as compared to $J 1$ allows excluding the competition of exchange interactions as a source of remanent magnetization at $T<$ $T_{\mathrm{N}}{ }^{42}$ Thus, the origin of strongly canted antiferromagnetic ground state in $\mathrm{Cu}_{3}(\mathrm{OH})_{2} \mathrm{~F}_{4}$ could be only Dzyaloshinski-Moriya (DM) interaction between $\mathrm{Cu} 1$ and $\mathrm{Cu} 2$ within trimerized $\mathrm{Cu} 2-\mathrm{Cu} 1$ - Cu2 chains. No alteration of DM component stemming from the intrinsic features of the crystal lattice in $\mathrm{Cu}_{3}(\mathrm{OH})_{2} \mathrm{~F}_{4}$ results in unusually large spontaneous magnetization. At $T<T_{\mathrm{C}}$, the remanence $M_{\mathrm{R}}$ constitutes large portion of saturation magnetization $M_{\mathrm{S}}$ which allows defining the canting angle $\varphi=4^{\circ}$ within trimerized chains of edge- and corner-sharing $\mathrm{Cu}(\mathrm{OH})_{2} \mathrm{~F}_{4}$ polyhedra.

\section{AUTHOR INFORMATION}

Corresponding Author

*E-mail: vasil@mig.phys.msu.ru

\section{ACKNOWLEDGEMENTS}

We are grateful to J.-Y. Lin for the useful comments. This work was supported by the Ministry of Education and Science of the Russian Federation in the framework of Increase Competitiveness Program of NUST "MISiS” grant K2-2017-084, by acts 211 of the Government of Russian Federation, Contracts No. 02.A03.21.0004, 02.A03.21.0006 and 02.A03.21.0011. Support by Russian Foundation for Basic Research through grants 16-02-00021 and 17-0200211 is acknowledged. Theoretical calculations and ESR measurements were supported by the Russian Science Foundation through the project 17-12-01207. This work was partially performed at Swiss Muon Source (S $\mu$ S), PSI, Villigen. S.H. acknowledges financial support by the Swiss National Science Foundation (SNF-Grant 200021_159736). 


\section{REFERENCES}

(1) R. Rückamp, J. Baier, M. Kriener, M.W. Haverkort, T. Lorenz, G.S. Uhrig, L. Jongen, A. Möller, G. Meyer, M. Grüninger, Zero-field incommensurate spin-Peierls phase with interchain frustration in TiOCl. Phys. Rev. Lett. 95 (2005) 097203.

(2) A. Seidel, C.A. Marianetti, F.C. Chou, G. Ceder, P.A. Lee, S=1/2 chains and spin-Peierls transition in TiOCl. Phys. Rev. B 67 (2003) 020405.

(3) A. Klümper, The spin-1/2 Heisenberg chain: thermodynamics, quantum criticality and spin-Peierls exponents. Eur. Phys. J. B 5 (1998) $677-685$.

(4) S. van Smaalen, L. Palatinus, A. Schnöbler, Incommensurate interactions and nonconventional spin-Peierls transition in TiOBr. Phys. Rev. B 72 (2005) 020105.

(5) J. Zhang, A. Wölfel, L. Li, S. van Smaalen, H.L. Williamson, R.K. Kremer, Magnetoelastic coupling in the incommensurate antiferromagnetic phase of FeOCl. Phys. Rev. B 80 (2012) 134428.

(6) H. Ben Yahia, M. Shikano, M. Tabuchi, H. Kobayashi, M. Avdeev, T. Tan, C.D. Ling, Synthesis and characterization of the crystal and magnetic structures and properties of the hydroxyfluorides $\mathrm{Fe}(\mathrm{OH}) \mathrm{F}$ and $\mathrm{Co}(\mathrm{OH}) \mathrm{F}$. Inorg. Chem. 53 (2013) 365 - 374.

(7) I. Danilovich, A. Merkulova, A. Polovkova, E. Zvereva, Y. Ovchenkov, I. Morozov, B. Rahaman, T. Saha-Dasgupta, C. Balz, H. Luetkens, O. Volkova, A. Shakin, A. Vasiliev, Vehement competition of multiple superexchange interactions and peculiar magnetically disordered state in $\mathrm{Cu}(\mathrm{OH})$ F. J. Phys. Soc. Jpn. 85 (2016) 024709.

(8) Y. Cudennec, A. Riou, Y. Gérault, A. Lecerf, Synthesis and crystal structures of Cd (OH) $\mathrm{Cl}$ and $\mathrm{Cu}(\mathrm{OH}) \mathrm{Cl}$ and relationship to brucite type. J. Sol. St. Chem. 151 (2000) $308-$ 312.

(9) M. Hagihala, X.G. Zheng, T. Toriyi, T. Kawae, Antiferromagnetism and geometric frustration in tetrahedral lattice hydroxyhalides $\mathrm{M}_{2}(\mathrm{OH})_{3} X$. J. Phys.: Condens. Matter 19 (2007) 145281. 
(10) M. Fujihala, M. Hagihala, X.G. Zheng, T. Kawae, Antiferromagnetic magnetic transition and spin fluctuations in the deformed pyrochlore compound $\beta-\mathrm{Fe}_{2}(\mathrm{OH})_{3} \mathrm{Cl}$. Phys. Rev. B 82 (2010) 024425.

(11) X.G. Zheng, T. Kawae, H. Yamada, K. Nishiyama, C.N. Xu, Coexisting ferromagnetic order and disorder in a uniform system of hydroxyhalide $\mathrm{Co}_{2}(\mathrm{OH})_{3} \mathrm{Cl}$. Phys. Rev. Lett. 97 (2006) 247204.

(12) T. Saha-Dasgupta, R. Valenti, H. Rosner, C. Gros, TiOCl, an orbital-ordered system? Europhys. Lett. 67 (2004) $63-69$.

(13) V. Kataev, J. Bajer, A. Möller, L. Jongen, G. Meyer, A. Freimuth, Orbital order in the low-dimensional quantum spin system TiOCl probed by ESR. Phys. Rev. B 68 (2003) 140405.

(14) P. Lemmens, K.Y. Choi, R. Valenti, T. Saha-Dasgupta, E. Abel, Y.S. Lee, F.C. Chou, Spin gap formation in the quantum spin systems TiOX, $\mathrm{X}=\mathrm{Cl}$ and Br. New J. Phys. 7 (2005) 74.

(15) A. Wiedenmann, J. Rossat - Mignot, J.P. Venien, P. Palvadeau, Two-dimensional behaviour and magnetic ordering in the layered compound VOCl. J. Magn. Magn. Mater. 45 (1984) $275-278$.

(16) A.C. Komarek, T. Taetz, M.T. Fernandes-Diaz, D.M. Trots, A. Möller, M. Braden, Strong magnetoelastic coupling in $\mathrm{VOCl}$ : Neutron and synchrotron powder $\mathrm{x}$-ray diffraction study. Phys. Rev. B 79 (2009) 104425.

(17) J. Angelkort, A. Wölfel, A. Schönleber, S. van Smaalen, R.K. Kremer, Strong magnetoelastic coupling in $\mathrm{VOCl}$ : Neutron and synchrotron powder $\mathrm{x}$-ray diffraction study. Phys. Rev. B 80 (2009) 144416.

(18) R.W. Grant, Magnetic structure of FeOCl. J. Appl. Phys. 42 (1971) 1619 - 1620.

(19) T. Pungas, The microscopic magnetic model of $\mathrm{Cu}(\mathrm{OH}) \mathrm{Cl}$. Master's Thesis, Tartu (2014). 
(20) X.G. Zheng, M. Hagihala, K. Nishiyama, T. Kawae, Exotic antiferromagnetic transition in deformed pyrochlore lattice $\mathrm{Ni}_{2}(\mathrm{OH})_{3} \mathrm{Cl}$ of atacamite-structure Physica B Condens. Matter 404 (2009) $677-679$.

(21) D. Tsuneishi, M. Ioki, H. Kawamura, Novel ordering of the pyrochlore Heisenberg antiferromagnet with the ferromagnetic next-nearest-neighbour interaction. J. Phys. Condens. Matter 19 (2007) 145273.

(22) X.G. Zheng, T. Kawae, Y. Kashitani, C.S. Li, N. Tateiwa, K. Takeda, H. Yamada, C.N. $\mathrm{Hu}, \mathrm{Y}$. Ren, Unconventional magnetic transitions in the mineral clinoatacamite $\mathrm{Cu}_{2} \mathrm{Cl}(\mathrm{OH})_{3}$. Phys. Rev. B 71 (2005) 052409.

(23) X.G. Zheng, T. Mori, K. Nishiyama, W. Higemoto, H. Yamada, K. Nishikubo, C.N. Xu, Antiferromagnetic transitions in polymorphous minerals of the natural cuprates atacamite and botallackite $\mathrm{Cu}_{2} \mathrm{Cl}(\mathrm{OH})_{3}$. Phys. Rev. B 71 (2005) 174404.

(24) H.O. Jeschke, F. Salvat-Pujol, E. Gati, N.H. Hoang, B. Wolf, M. Lang, J.A. Schlueter, R. Valentí, Barlowite as a canted antiferromagnet: Theory and experiment. Phys. Rev. B 92 (2015) 094417.

(25) L. Zhao, M.T. Fernandez-Diaz, L.H. Tjeng, A.C. Komarek, Oxyhalides: A new class of high-TC multiferroic materials. Sci. Adv. 2 (2016) 1600353.

(26) G. Giester and E. Libowitzky, Crystal structures and Raman spectra of $\mathrm{Cu}(\mathrm{OH}) \mathrm{F}$ and $\mathrm{Cu}_{3}(\mathrm{OH})_{2} \mathrm{~F}_{4}$ Z. Kristallogr. 218 (2003) $351-356$.

(27) I.L. Danilovich, O.S. Volkova, A.N. Vasiliev, Magnetism of polyanionic compounds of transition metals. Low Temp. Phys. 43 (2017) $529-542$.

(28) A. Suter, B.M. Wojek, Musrfit: A free platform-independent framework for $\mu$ SR data analysis, Phys. Procedia 30 (2012) 69-73.

(29) P. Giannozzi, S. Baroni, N. Bonini, et al., QUANTUM ESPRESSO: a modular and opensource software project for quantum simulations of materials. J. Phys.: Condens. Matter 21 (2009) 395502. 
(30) J. P. Perdew, K. Burke, M. Ernzerhof, Generalized gradient approximation made simple. Phys. Rev. Lett. 77 (1996) $3865-3868$.

(31) G. Kresse and J. Furthmuller, Efficient iterative schemes for ab initio total-energy calculations using a plane-wave basis set. Phys. Rev. B 54 (1996) 11169 - 11186.

(32) H. J. Xiang, E. J. Kan, Su-Huai Wei, M.-H. Whangbo, X. G. Gong, Predicting the spinlattice order of frustrated systems from first principles. Phys. Rev. B 84 (2011) 224429.

(33) A. I. Liechtenstein, V. I. Anisimov, J. Zaanen, Density-functional theory and strong interactions: Orbital ordering in Mott-Hubbard insulators. Phys. Rev. B 52 (1995) R5467 R5470.

(34) J.B. Goodenough, An interpretation of the magnetic properties of the perovskite-type mixed crystals $\mathrm{La}_{1-x} \mathrm{Sr}_{x} \mathrm{CoO}_{3-\lambda}$. J. Phys. Chem. Sol. 6 (1958) $287-297$.

(35) J. Kanamori, Superexchange interaction and symmetry properties of electron orbitals. J. Phys. Chem. Sol. 10 (1959) $87-98$.

(36) R.M. Krishna, S.K. Gupta, Electron paramagnetic resonance investigations of the $\mathrm{Cu}^{2+}$ ion in a variety of host lattices-A review. Bull. Magn. Res. 16 (1994) 239 - 291.

(37) K. V. Zakharov, E. A. Zvereva, P. S. Berdonosov, E. S. Kuznetsova, V. A. Dolgikh, L. Clark, C. Black, P. Lightfoot, W. Kockelmann, Z. V. Pchelkina, S. V. Streltsov, O. S. Volkova, A. N. Vasiliev, Thermodynamic properties, electron spin resonance, and underlying spin model in $\mathrm{Cu}_{3} \mathrm{Y}\left(\mathrm{SeO}_{3}\right)_{2} \mathrm{O}_{2}$ Cl. Phys. Rev. B 90 (2014) 214417.

(38) Y. Yasui, Y. Kawamura, Y. Kobayashi, M. Sato, Magnetic and dielectric properties of one-dimensional array of $\mathrm{S}=1 / 2$ linear trimer system $\mathrm{Na}_{2} \mathrm{Cu}_{3} \mathrm{Ge}_{4} \mathrm{O}_{12}$. J. Appl. Phys. 115 (2014) $17 \mathrm{E} 125$.

(39) J.H. Brewer, S.R. Kreitzman, D.R. Noakes, E.J. Ansaldo, D.R. Harshman, and R. Keitel, Observation of muon-fluorine" hydrogen bonding" in ionic crystals. Phys. Rev. B 33 (1986) $7813-7816$. 
(40) A. Yaouanc and P. Dalmas de Rotier, Muon Spin Rotation, Relaxation and Resonance, Oxford University Press, Oxford, U.K. (2011).

(41) A.A. Mostofi, J.R. Yates, G. Pizzi, Y.S. Lee, I. Souza, D. Vanderbilt, N. Marzari, An updated version of wannier90: A tool for obtaining maximally-localised Wannier functions. Comput. Phys. Commun. 185 (2014) 2309 - 2310.

(42) S.L. Drechsler, J. Richter, R. Kuzian, et. al., Helimagnetism and weak ferromagnetism in edge-shared chain cuprates. J. Magn. Magn. Mater. 316 (2007) 306 - 312. 